\title{
STUDY ON GRANULARITY DISTRIBUTION OF POWDER BY FRACTAL MODELS
}

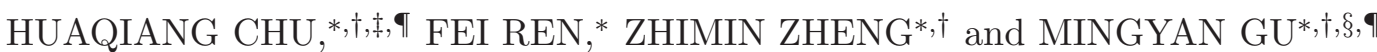 \\ ${ }^{*}$ School of Energy and Environment \\ Anhui University of Technology, Ma'anshan 243002, P. R. China \\ ${ }^{\dagger}$ Key Laboratory of Metallurgical Emission Reduction and Resources Recycling \\ Anhui University of Technology, Ministry of Education \\ Ma'anshan 243002, P. R. China \\ †hqchust@163.com \\ §mingyan_gu@qq.com
}

Received January 10, 2017

Revised April 14, 2017

Accepted April 17, 2017

Published June 2, 2017

\begin{abstract}
The granularity distribution of powder is shown to have the fractal characters. Based on the fractal theory, three algebraic expressions for the fractal dimension, the cumulative probability and the mass distribution of particles were obtained. Three expressions are the functions of the concentration of particles, the diameter of particles, the maximum and the minimum particle diameters. No additional empirical constant is introduced in these expressions. These fractal models contain less empirical constants than the conventional correlations. The fractal behavior about coal particles was discussed in detail. Simulations were also performed by fractal Monte Carlo technique. The proposed technique may provide us a new approach to analyze the granularity distribution of powder.
\end{abstract}

Keywords: Powder; Granularity Distribution; Fractal.

\footnotetext{
${ }^{\top}$ Corresponding authors.

This is an Open Access article published by World Scientific Publishing Company. It is distributed under the terms of the Creative Commons Attribution 4.0 (CC-BY) License. Further distribution of this work is permitted, provided the original work is properly cited.
} 


\section{INTRODUCTION}

Particles size, such as coal powder and soot, is an important parameter for industry analysis and has significant effects on the behaviors of powder distribution. Havlin and Ben-Avraham ${ }^{1}$ showed that the size distribution of particles exhibited some kind of self-similarity. The concept of fractal was proposed by Mandelbrot.2 Avnir et al. ${ }^{[3}$ extended the fractal method to study the coal behaviors. The results ${ }^{4}$ indicated that the coal and rock showed the fractal behavior over the range from atom scale to grain size. Carpinteri et al! $\frac{5] 6}{6}$ explained the scaling laws for strength and toughness in the breaking behavior of disordered materials by using the fractal and multifractal approaches. Engleman et al..$^{7}$ demonstrated that the number-size distribution followed a fractal law for fragments which are not too large. Although a number of fractal models have been developed, ${ }^{811}$ these models fail to predict the fragment size distribution. Recently, some investigators ${ }^{12[13}$ studied the fragments from physical and geophysical points of view, and a multifractality was also developed 14 [16

According to the results of $\mathrm{Xie}^{4}$ and $\mathrm{Yu}$ et al., 17 the cumulative distribution of particles with diameter smaller than $\lambda$ satisfies the fractal scaling law:

$$
Y_{w}(\lambda) \propto \lambda^{3-D_{f}},
$$

where $\lambda$ is the diameter of particles, $Y_{w}(\lambda)$ is the accumulative weight percentage and $D_{f}$ is the fractal dimension. If there is a linear relationship between the accumulative weight percentage and the grain size in log-log plot, it indicates that the accumulative weight percentage of powder has the fractal structure. Let the slope be $k$, the fractal dimension can be written as

$$
D_{f}=3-k .
$$

Equation (2) is only a function of the particles size, independent of other parameters. In fact, the fractal dimension is consistent with the accumulative weight percentage. In Ref. 17, the fractal dimension was obtained by the least square method. Jiang et al ${ }^{18}$ obtained the polynomial expression for the fractal dimension by employing the binary regression analysis, but the expression had several empirical constants.

In this work, we attempt to derive new analytical expressions for the fractal dimension, the cumulative probability of size and the mass distributions for particles. This paper is organized as follows. In
Sec. 2, a fractal analysis is presented. Then, the cumulative probability of particle and the mass distribution are both deduced in Sec. 3. In Sec. 4, the random particle sizes are simulated based on the fractal Monte Carlo method. Finally, concluding remarks are included in Sec. 5

\section{FRACTAL ANALYSIS OF GRAIN SIZES}

According to fractal geometry theory, the cumulative size-distribution of particles or islands (on earth) , $N$, has been proven to follow the fractal scaling law 2 :

$$
N(L \geq \lambda)=\left(\frac{\lambda_{\max }}{\lambda}\right)^{D_{f}},
$$

where $L$ is a certain local range of length scales, $\lambda$ is the diameter of particles, $\lambda_{\max }$ is the maximum particle diameter, $N$ is the cumulative population of particles whose sizes are greater than or equal to $\lambda$, and $D_{f}$ is the fractal dimension for particles, with $1<D_{f}<2$ in two dimensions and $2<D_{f}<3$ in three dimensions. It is evident that the total number of particles, from the smallest diameter to the largest diameter, can be obtained from Eq. (3) as

$$
N_{t}\left(L \geq \lambda_{\min }\right)=\left(\frac{\lambda_{\max }}{\lambda_{\min }}\right)^{D_{f}} .
$$

Differentiating on both sides of Eq. (3) results in the number of particles whose sizes are within the infinitesimal range of $\lambda$ to $\lambda+d \lambda$

$$
-d N=D_{f} \lambda_{\max }^{D_{f}} \lambda^{-\left(D_{f}+1\right)} d \lambda .
$$

In Eq. (5), $-d N>0$, which implies that the number of particles decreases with the increase of particle size.

Combining Eqs. (44) and (5), we can obtain

$$
-\frac{d N}{N_{t}}=D_{f} \lambda_{\min }^{D_{f}} \lambda^{-\left(D_{f}+1\right)} d \lambda=f(\lambda) d \lambda
$$

where $f(\lambda)=D_{f} \lambda_{\min }^{D_{f}} \lambda^{-\left(D_{f}+1\right)}$ is the probability density function.20 In general, the ratio of $\lambda_{\min } / \lambda_{\max }<10^{-2}$. In this work, we choose $\lambda_{\min } / \lambda_{\max } \sim 10^{-3}$ as the ratio of particles. Thus, $\left(\frac{\lambda_{\min }}{\lambda_{\max }}\right)^{D_{f}} \cong 0$ since $2<D_{f}<3$ in three dimensions.

Fractal dimension is a basic parameter in fractal theory. The expression of the fractal dimension is defined as $D_{f}=-\lim _{\lambda \rightarrow 0} \frac{\ln N(\lambda)}{\ln \lambda}$, which may be 
Table 1 The Distribution of Soot and Its Fractal Dimension (Wuhan N219)! 17

\begin{tabular}{ccccccc}
\hline Size $(\boldsymbol{\mu m})$ & $<\mathbf{1 0}$ & $<\mathbf{3 0}$ & $<\mathbf{5 0}$ & $<\mathbf{6 0}$ & $<\mathbf{7 0}$ & $\boldsymbol{D}_{\boldsymbol{f}}$ \\
\hline$\phi$ & 22.18 & 37.27 & 79.95 & 88.22 & 100 & 2.20 (Ref. [17) \\
$\ln \phi$ & -1.506 & -0.987 & -0.224 & -0.125 & 0 & 2.20 (Eq. 7) \\
$\ln \frac{\lambda_{\min }}{\lambda_{\max }}$ & -4.605 & -5.714 & -6.215 & -6.377 & -6.571 & \\
\hline
\end{tabular}

obtained by the slope of the log-log plot. In present work, the fractal dimension $D_{f}$ is determined by ${ }^{20}$

$$
D_{f}=d-\frac{\ln \phi}{\ln \left(\lambda_{\min } / \lambda_{\max }\right)},
$$

where $d$ is the Euclidean dimension, and $d=2(3)$ in two (three) dimensions, $\phi$ is the concentration of particles.

The average diameter of particles can be found from the statistical property of fractal media, and it is

$$
\lambda_{\mathrm{av}}=\int \lambda f(\lambda) d \lambda=\lambda_{\min } \frac{D_{f}}{D_{f}-1} .
$$

In Eq. (8), the condition $\left(\frac{\lambda_{\min }}{\lambda_{\max }}\right)^{D_{f}} \cong 0$ has been applied.

Let $k^{\prime}=\ln \phi / \ln \left(\lambda_{\min } / \lambda_{\max }\right)$, Eq. (7) can be rewritten as

$$
D_{f}=d-k^{\prime},
$$

where $k^{\prime}$ has the clear physical meaning, while in Eq. (2) $k$ only represents a slope and the physical meaning behind the constant $k$ is not revealed.

According to Eq. (7), the fractal dimension for the soot particles (combustion products) can be obtained. The distribution of Wuhan N219 soot was given in Ref. 17, as shown in Table 1. According to the fractal model, the soot fractal dimension was calculated by Eq. (7), which also was gave in Table 1. Figure 1 showed the relationship between $\ln \left(\lambda_{\min } / \lambda_{\max }\right)$ and $\ln \phi$. From Fig. 1, it is found that there is a linear relationship between the accumulative weight percentage and the grain size in log-log plot, so the accumulative weight percentage of soot particles has the fractal structure.

The similar format to that from Eq. (77) can be obtained from Eq. (11). In Eq. (7), $D_{f}$ is the fractal dimension for the particles whose sizes are greater than or equal to $\lambda$, whereas $D_{f}$ in Eq. (1) is for the particles with diameter lesser than or equal to $\lambda$. The two equations are essentially equivalent, but Eq. (17) is easily applied to obtain the fractal dimension and can be applied to two dimensions and three dimensions.

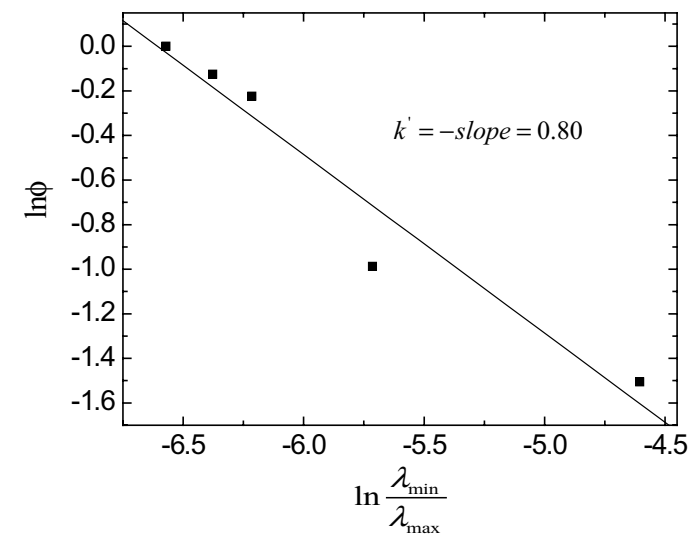

Fig. $1 \ln \left(\lambda_{\min } / \lambda_{\max }\right)$ versus $\ln \phi$.

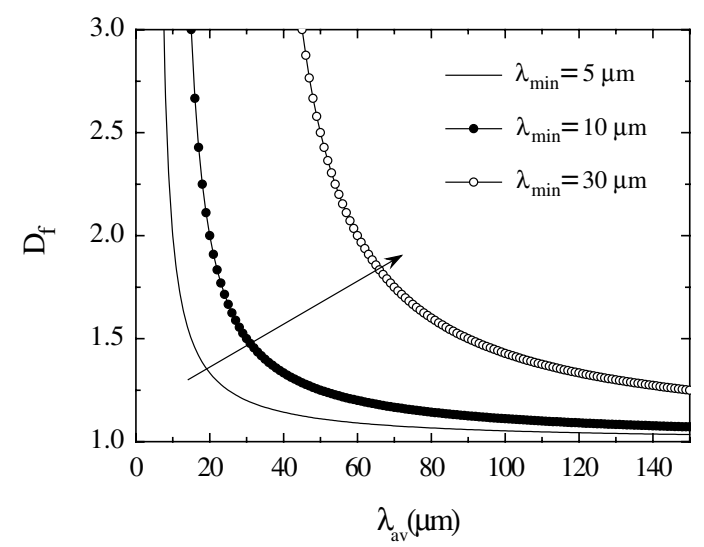

Fig. 2 The fractal dimension of soot particles versus average size.

According to Eqs. (7), (8) and Table 1 the fractal dimension of soot particles and average size and the accumulative weight percentage are plotted in Figs. 2 and 3. Figure 2 indeed shows the fractal dimension increases with the decrease of the mean diameter using Eq. (8) by assuming $\lambda_{\min }=$ $5,10,30 \mu \mathrm{m}$, respectively. From Fig. 3 , it is seen that the fractal dimension is rising with the increase of the accumulative weight percentage and it will be equal to three when the accumulative weight

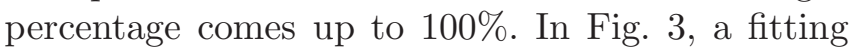
polynomial was also given about the concentration of particles $\phi$ and the fractal dimension $D_{f}$. If the concentration of particles is known, it is easy 


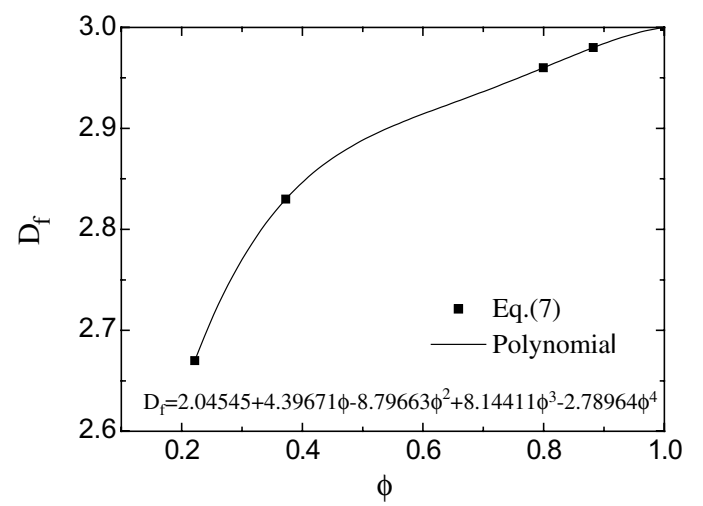

Fig. 3 The fractal dimension of soot particles versus the accumulative weight percentage.

to obtain the fractal dimension using this fitting polynominal.

\section{FRACTAL ANALYSIS OF PARTICLE SIZE AND MASS DISTRIBUTIONS}

In order to verify the above analysis, we will investigate the size distribution and the mass distribution for coal powder. In general, the diameters of particles are less than $500 \mu \mathrm{m}$. The analysis of grain sizes for coal powder is very important in industrial application.

The definition of the cumulative probability in the range of $\lambda_{\min } \sim \lambda$ is often given by

$$
R(\lambda)=\frac{N(\lambda)}{N_{t}} \text {. }
$$

Submitting Eqs. (3) and (4) into Eq. (10), we can obtain

$$
\begin{aligned}
R(\lambda) & =\frac{N(\lambda)}{N_{t}}=\frac{\int_{\lambda_{\min }}^{\lambda}-D_{f} \lambda_{\max }^{D_{f}} \lambda^{-\left(D_{f}+1\right)} d \lambda}{\int_{\lambda_{\min }}^{\lambda_{\max }}-D_{f} \lambda_{\max }^{D_{f}} \lambda^{-\left(D_{f}+1\right)} d \lambda} \\
& =\frac{\lambda^{-D_{f}}-\lambda_{\min }^{-D_{f}}}{\lambda_{\max }^{-D_{f}}-\lambda_{\min }^{-D_{f}}}=1-\left(\frac{\lambda_{\min }}{\lambda}\right)^{D_{f}} .
\end{aligned}
$$

Equation (11) implies that $R=0$ as $\lambda \rightarrow \lambda_{\min }$, and $R=1$ as $\lambda \rightarrow \lambda_{\max }$. Thus $R$ is in the range of $0-1$ and approximately corresponds to a set of random numbers of $0-1$.

Similarly, the mass distribution can be given as

$$
\begin{aligned}
M(\lambda) & =\frac{\int_{\lambda_{\min }}^{\lambda}-\psi \rho \lambda^{3} D_{f} \lambda_{\max }^{D_{f}} \lambda^{-\left(D_{f}+1\right)} d \lambda}{\int_{\lambda_{\min }}^{\lambda_{\max }}-\psi \rho \lambda^{3} D_{f} \lambda_{\max }^{D_{f}} \lambda^{-\left(D_{f}+1\right)} d \lambda} \\
& =\frac{\lambda^{3-D_{f}}-\lambda_{\min }^{3-D_{f}}}{\lambda_{\max }^{3-D_{f}}-\lambda_{\min }^{3-D_{f}}}=\left(\frac{\lambda}{\lambda_{\max }}\right)^{3-D_{f}},
\end{aligned}
$$

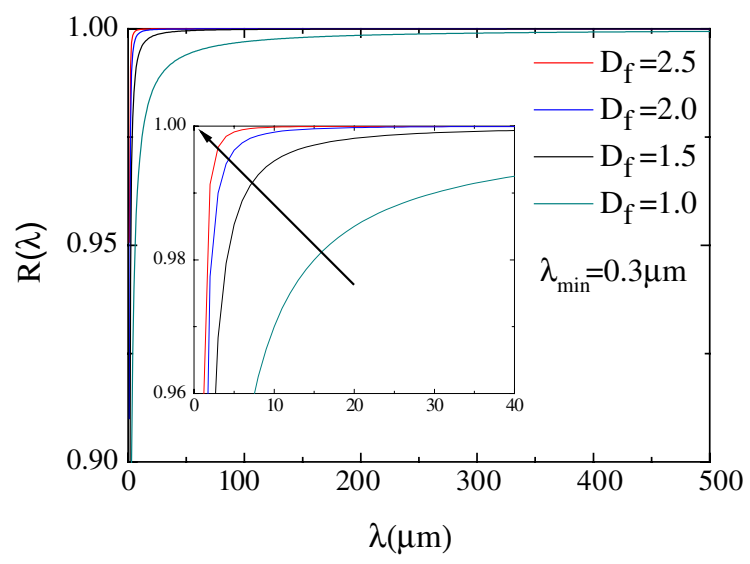

(a)

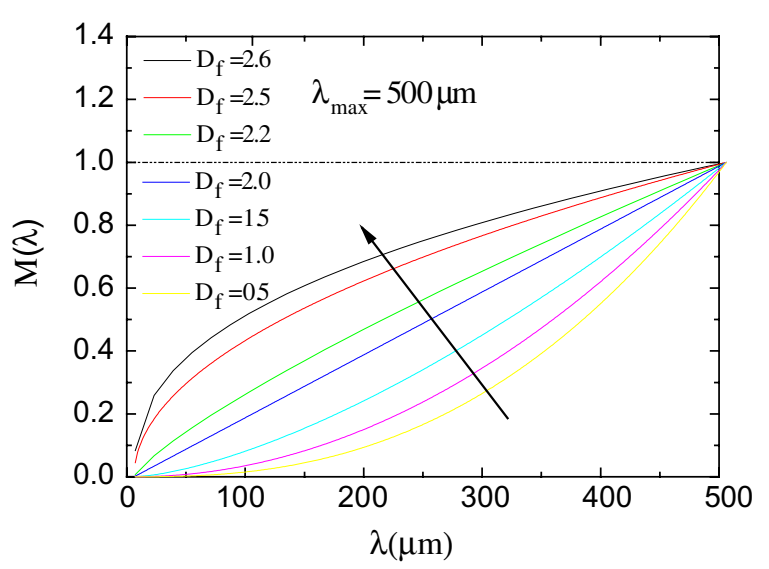

(b)

Fig. 4 The effect of coal powder sizes on (a) the cumulative probability, and (b) the mass distribution at different fractal dimensions.

where $\psi$ is the shape factor and $\rho$ is the density of coal powder. In the above two equations, we apply the assumptions: $\left(\lambda_{\min } / \lambda_{\max }\right)^{D_{f}} \cong 0$ and $\left(\lambda_{\min } / \lambda_{\max }\right)^{3-D_{f}} \cong 0$.

Figure 4 gives the effect of coal powder sizes on the cumulative probabilities and the mass distributions at different fractal dimensions. From Fig. 4(a), it shows that the diameters of most particles are less than $200 \mu \mathrm{m}$. In addition, the figure indicates that the bigger the fractal dimension, the more finesse and uniform of particles are present. Therefore, we can obtain the fine coal particles by controlling the fractal dimension. It is also interestingly to be found from Fig. 4 (b) that when $D_{f}=2, M(\lambda) \sim \lambda$ is linear and the mass distribution of differently sized coal particles is the same (i.e. with the same probability). When $0<D_{f}<2$, the bigger coal particles are dominant, and when $2<D_{f}<3$, the smaller particles are dominant. 


\section{SIMULATIONS BY THE FRACTAL MONTE CARLO TECHNIQUE}

In this section, the random particle sizes by Monte Carlo technique based on the fractal theory are simulated.

If the average diameter is known, the minimum diameters of particles can be determined by Eq. (8)

$$
\lambda_{\min }=\frac{D_{f}-1}{D_{f}} \lambda_{\mathrm{av}} .
$$

Due to Eq. (11), we can obtain

$$
1-R=\left(\frac{\lambda_{\min }}{\lambda}\right)^{D_{f}} .
$$

Based on the above equations, a particle size $\lambda$ can be expressed as

$$
\lambda=\frac{\lambda_{\min }}{(1-R)^{1 / D_{f}}}=\frac{D_{f}-1}{D_{f}(1-R)^{1 / D_{f}}} \lambda_{\mathrm{av}}
$$

where $\lambda_{\min }<\lambda<\lambda_{\max }$. Equation (15) is a probability model for diameter of particles in the present simulation. For the $i$ th particle chosen randomly, Eq. (15) can be rewritten as

$$
\lambda_{i}=\frac{\lambda_{\min }}{\left(1-R_{i}\right)^{1 / D_{f}}}=\frac{D_{f}-1}{D_{f}\left(1-R_{i}\right)^{1 / D_{f}}} \lambda_{\mathrm{av}},
$$

where $i=1,2,3, \ldots, J$, and $J$ is the total number of Monte Carlo simulations in one run for a given average diameter and an accumulative weight percentage. $R_{i}$ is a random number of $0-1$ produced by computer. Equation (16) gives an explicit probability expression for particle size distribution. In this equation, the fractal dimension is also included.

The average diameter of the particles calculated in the present fractal Monte Carlo simulations is given by $\mathrm{Yu}$ et al $!^{21}$ and Feng et al. $!^{22}$

$$
\bar{\lambda}=\frac{1}{J} \sum_{i=1}^{J} \lambda_{i} .
$$

Following Feng et al., 22 the convergence criterion is employed as

$$
\bar{\lambda}=\frac{1}{J} \sum_{i=1}^{J} \lambda_{i} \geq \lambda_{\mathrm{av}} .
$$

When Eq. (18) is satisfied, the simulation will stop and the particle sizes are recorded.

The algorithm for the random particles sizes is summarized as follows:

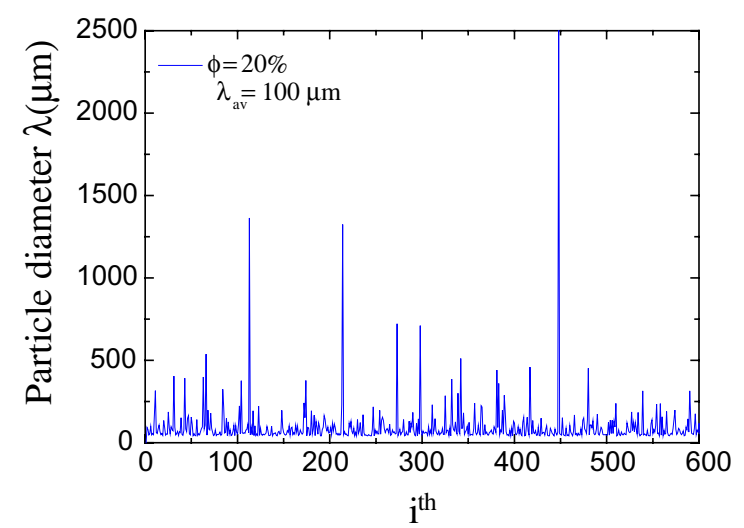

(a)

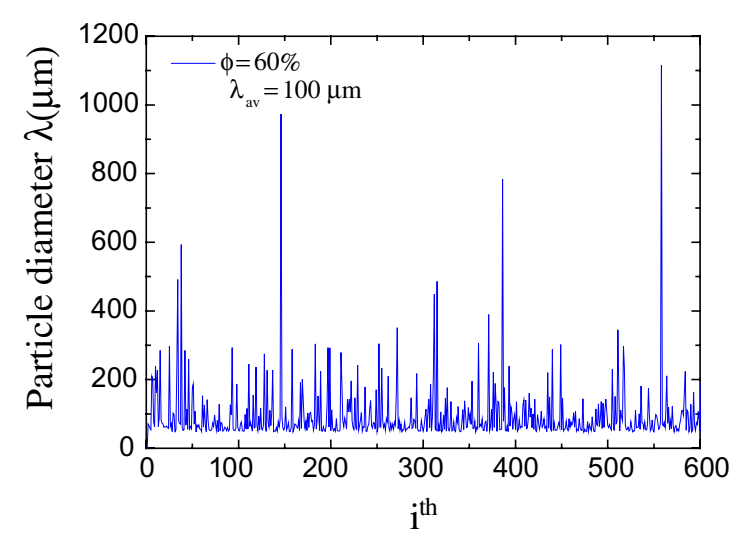

(b)

Fig. 5 The particle sizes simulated by the fractal Monte Carlo simulations.

(1) Given an average diameter $\lambda_{\mathrm{av}}$ in experiments and an accumulative weight percentage $\phi$.

(2) Find $D_{f}$ from Eq. (7), $\lambda_{\min }, \lambda_{\max }$ from Eq. (13).

(3) Produce a random number $R_{i}$ of $0-1$ by computer.

(4) Calculate $\lambda_{i}$ from Eq. (16).

Procedures (1)-(4) are repeated for calculation of the particle size until Eq. (18) is satisfied.

Figure 5 gives the particle sizes randomly chosen by the present model (Eq. (16). From Fig. 5, it is found that the number of larger particles is much less than that of smaller particles, which is qualitatively consistent with the fractal theory. This simulation process is also conducted by other authors. 23.25

\section{CONCLUSIONS}

In this paper, both the fractal theory and the Monte Carlo technique are employed to derive new analytical expressions. According to the fractal theory, the algebraic expression for the fractal dimension, 
together with the expressions of cumulative probability of particles and the mass distribution has been obtained. Compared to the conventional correlations, these resulting expressions, which are the functions of the concentration of particles, the diameter of particles, the maximum particle diameter and the minimum particle diameter, contain less empirical constants. Particularly, the fractal behavior about coal particles is discussed in detail, which indicates that one can obtain the fine coal particles by controlling the fractal dimension. In addition, simulations were also performed using the fractal Monte Carlo technique, with the results qualitatively consistent with the fractal theory. It is found that the number of larger particles is much less than that of smaller particles. The proposed technique may have the potential in analyzing other properties of particles.

\section{ACKNOWLEDGMENTS}

This work was supported by the National Natural Science Foundation of China (Nos. 51676002, 51376008, 51606001) and the Anhui Provincial Natural Science Foundation (No. 1408085QE100). The authors would like to thank Prof. Boming Yu for many useful discussions.

\section{REFERENCES}

1. S. Havlin and D. Ben-Avraham, Diffusion in disordered media, Adv. Phys. 36(6) (1987) 695-798.

2. B. B. Mandelbrot, The Fractal Geometry of Nature (W. H. Freemen, New York, 1982).

3. D. Avnir, D. Farin and P. Pfeifer, Chemistry in noninteger dimensions between two and three. II. Fractal surfaces of adsorbents, J. Chem. Phys. 79(7) (1983) 3566-3571.

4. H. P. Xie, Fractals in Rock Mechanics (Balkema Publishers, The Netherlands, 1993).

5. A. Carpinteri and N. Pugno, A multifractal comminution approach for drilling scaling laws, Powder Technol. 131 (2003) 93-98.

6. A. Carpinteri and B. Chiaia, Multifractal scaling laws in the breaking behavior of disordered materials, Chaos Solitons Fractals 8 (1997) 135-150.

7. R. Engleman, N. Rivier and Z. Jaeger, Size distribution in sudden breakage by the use of entropy maximization, J. Appl. Phys. 63 (1988) 4766-4768.

8. M. Matsushita, Fractal viewpoint of fracture and accretion, J. Phys. Soc. Japan 54 (1985) 857-860.
9. D. L. Turcotte, Fractals and fragmentation, J. Geophys. Res. 91 (1986) 1921-1926.

10. M. Rieu and G. Sposito, Fractal fragmentation, soil porosity, and soil water properties. I. Theory, Soil Sci. Soc. Am. J. 55 (1991) 1231-1238.

11. E. Perfect, Fractal models for the fragmentation of rocks and soils: A review, Eng. Geol. 48 (1997) 185198.

12. E. Ben-Naim and P. L. Krapivsky, Fragmentation with a steady source, Phys. Lett. A 48 (2000) 275278.

13. P. Hyslip and L. E. Vallejo, Fractal analysis of the roughness and size distribution of granular materials, Eng. Geol. 48 (1997) 231-244.

14. O. Sotolongo-Costa, A. H. Rodriguez and G. J. Rodgers, Dimensional crossover in fragmentation, Physica A 286 (2000) 638-642.

15. E. S. C. Ching, Multifractality of mass distribution in fragmentation, Physica A 288 (2000) 402-408.

16. C. A. Hecht, Appolonian packing and fractal shape of grains improving geomechanical properties in engineering geology, Pure Appl. Geophys. 157 (2000) 487-504.

17. K. Yu and Z. S. Zheng, Fractal characteristics on granularity distribution of powder, Chin. J. Mater. Res. 9(6) (1995) 536-542 (in Chinese).

18. X. M. Jiang, H. P. Yang, Y. Li and H. Liu, Fractal characteristics of coal powder, J. China Coal Soc. 28(4) (2003) 414-418 (in Chinese).

19. B. M. Yu and P. Cheng, A fractal model for permeability of bi-dispersed porous media, Int. J. Heat Mass Transf. 45(13) (2002) 2983-2993.

20. B. M. Yu and J. H. Li, Some fractal characters of porous media, Fractals 9(3) (2001) 365-372.

21. B. M. Yu, M. Q. Zou and Y. J. Feng, Permeability of fractal porous media by Monte Carlo simulations, Int. J. Heat Mass Transf. 48(13) (2005) 2787-2794.

22. Y. J. Feng, B. M. Yu, K. M. Feng, P. Xu and M. Q. Zou, Thermal conductivity of nanofluids and size distribution of nanoparticles by Monte Carlo simulations, J. Nanopart. Res. 10 (2008) 1319-1328.

23. Q. Xu, B. M. Yu, J. C. Cai, Dielectric constant of porous ultra low- $k$ dielectrics by fractal-Monte Carlo simulations, Phys. Lett. A 373(22) (2009) 19781982.

24. B. Q. Xiao, G. P. Jiang, Y. Yang and D. M. Zheng, Prediction of convective heat transfer of nanofluids based on fractal-Monte Carlo simulations, Int. J. Mod. Phys. C 24(1) (2013) 1250090.

25. J. C. Cai, X. Y. Hu, B. Q. Xiao, Y. F. Zhou and W. Wei, Recent developments on fractal-based approaches to nanofluids and nanoparticle aggregation, Int. J. Heat Mass Transf. 105 (2017) 623-637. 